\title{
Visual motion sensitivity in dyslexia: evidence for temporal and energy integration deficits
}

\author{
Joel B. Talcott*, Peter C. Hansen, Elikem L. Assoku, John F. Stein \\ University Laboratory of Physiology, Parks Road, Oxford OX1 3PT, UK
}

Received 12 January 1999; received in revised form 3 December 1999; accepted 14 December 1999

\begin{abstract}
In addition to poor literacy skills, developmental dyslexia has been associated with multisensory deficits for dynamic stimulus detection. In vision these deficits have been suggested to result from impaired sensitivity of cells within the retino-cortical magnocellular pathway and extrastriate areas in the dorsal stream to which they project. One consequence of such selectively reduced sensitivity is a difficulty in extracting motion coherence from dynamic noise, a deficit associated with both developmental dyslexia and persons with extrastriate, dorsal stream lesions. However the precise nature of the mechanism(s) underlying these perceptual deficits in dyslexia remain unknown. In this study, we obtained motion detection thresholds for 10 dyslexic and 10 control adults while varying the spatial and temporal parameters of the random dot kinematogram (RDK) stimuli. In Experiment 1 stimulus duration was manipulated to test whether dyslexics are specifically impaired for detecting short duration, rather than longer stimuli. Dot density was varied in Experiment 2 to examine whether dyslexics' reduced motion sensitivity was affected by the amount of motion energy present in the RDKs. Dyslexics were consistently less sensitive to coherent motion than controls in both experiments. Increasing stimulus duration did not improve dyslexics' performance, whereas increasing dot density did. Thus increasing motion energy assisted the dyslexics, suggesting that their motion detectors have a lower signal to noise ratio, perhaps due to spatial undersampling. (C) 2000 Elsevier Science Ltd. All rights reserved.
\end{abstract}

Keywords: Coherent motion; Visual; Temporal processing; Reading; Magnocellular; Dyslexia

\section{Introduction}

A large proportion of school age children and adults have unexpected difficulties acquiring competent reading skills $[47,50]$. This is often termed developmental dyslexia if their measured literacy skills are significantly lower than their other cognitive abilities ([2], cf. [54]). Although deficits in component language skills such as phonological awareness are recognised as important causes of poor reading $[8,49,55]$, there is now a large amount of literature showing that deficient

\footnotetext{
* Corresponding author. Tel.: +44-1865-272539; fax: +44-1865272469 .

E-mail address: joel.talcott@physiol.ox.ac.uk (J.B. Talcott).
}

visual processing skills are also associated with reading difficulties (see $[29,30,32,46,56]$ for review). In the laboratory, many developmental dyslexics are found to be less sensitive than controls to dynamic visual stimuli, especially those of low contrast, low luminance, low spatial frequencies, and high temporal frequencies $[10,28,30,31,33-35]$. Detection of such stimuli is likely to depend primarily upon the sensitivity of magnocells (M-cells) that comprise the retino-cortical M-pathway $[25,36,47]$. Detection of stimulus attributes such as high spatial frequencies, low temporal frequencies and colour differences depend more on the sensitivity of parvocells $[26,37,48]$. Sensitivity for these stimulus parameters are usually unimpaired in dyslexics $[10,28,31,33,34]$. Some psychophysical studies have failed to confirm that such visual deficits are associated 
with dyslexia ([20,21,61], see also, [17] for review). Nevertheless most experimental evidence is consistent with the hypothesis that the M (or transient) pathway is a locus of their subtle visual impairments (for reviews see $[29,30,56])$.

The primary retino-cortical visual stream consists of magno (M) and parvo (P) components, designated as such by the specificity of their projections to the dorsal laminae of the lateral geniculate nucleus (LGN) in the thalamus. In contrast to parvocells, cells of the Mstream have large cell bodies and thickly myelinated axons with rapid membrane dynamics and high conduction velocities dedicated to stimulus timing and the detection of rapid change. Receptive field sizes of $\mathrm{M}$ cells are larger than P-cells, a consequence of their greater spatial summation of pooled photoreceptor responses. As a result $\mathrm{M}$-cells are more sensitive to lower, rather than higher, spatial frequencies [13]. In cortex, M-cells provide the major input to the dorsal stream projecting from $\mathrm{V} 1$ to extrastriate areas such as V5/MT and the posterior parietal cortex [37,39]. Among other functions such as providing information about the spatial location of objects and guiding movements toward visual targets, dorsal stream areas mediate the processing of object motion $[5,14,22,36,38,51,57,59]$.

Firing rates of cells within MT can predict primates' responses to direction of motion in a psychophysical task $[9,40]$ and lesions to the same areas selectively reduce sensitivity to motion stimuli $[38,41,42]$. Lesions to the homologue of primate V5/MT and surrounding areas have also been shown to cause profound deficits in the ability to discriminate motion from dynamic noise in humans $[4,23,45,60,65,66]$. Visual tasks that tap the sensitivity of M-cells up to and including primary visual cortex are reported to be only slightly impaired in these same individuals $[4,23]$.

It has been shown in single unit studies in nonhuman primates that random dot kinematograms (RDK) provide a sensitive measure of $\mathrm{M}$-stream sensitivity $[9,40,41]$. In the typical RDK, a proportion of the total pixel elements, or 'dots', move coherently (i.e., their direction of motion is perfectly correlated over successive screen refreshes). The remaining noise dots move independently at the same speed, so that their directions of motion do not correlate over time. Lifetimes of single dots can be limited to prevent subjects from detecting motion by tracking the trajectory of a single dot. Thus, on every screen refresh, a certain percentage of dots disappear; they then reappear at random positions within the stimulus patch. Ability to see global coherent RDK motion then depends on the successful detection and integration of local motion signals over both space and time $[6,7,53,63]$, and the smallest proportion of dots that have to move coher- ently for the subject to perceive global motion gives the threshold for coherent motion detection.

Developmental dyslexics have been shown to be less sensitive than controls to motion stimuli both in psychophysical $[10,16,43,52,58,64]$ and neuroimaging $[12,15]$ studies. However the mechanism by which the putative M-pathway deficit results in disrupted motion perception is still unclear. For example, Walther-Müller [61] has suggested that any of a number of possible anomalies in either (or both) the temporal or spatial response functions of cells in the retino-cortical Mpathway could cause a deficit in dynamic stimulus detection. Dyslexics might also have poor integration of these signals at higher cortical levels. Either or both of these factors would decrease their ability to detect global coherent motion and would be consistent with anatomical evidence of structural impairment in both the retino-cortical M-pathway and within the extrastriate dorsal stream of dyslexics $[18,28,44]$. However, other studies have suggested that sensory deficits in dyslexia are associated more with a problem detecting short duration stimuli whether they are dynamic or not $([19,20]$ for review see [17]). Such a deficit would be difficult to reconcile with the hypothesis of a visual deficit specific to the M-stream.

In this study dyslexics' and controls' thresholds for coherent motion were obtained in two experiments in which the temporal and spatial parameters of the RDK stimuli employed were varied. These parameters were chosen because the percept of global coherent motion depends upon the adequate integration of both the spatially distributed motion signal provided by each dot and the global temporal integration of local motion signals between successive animation frames. In Experiment 1 stimulus duration was varied in two dot size conditions to test whether dyslexics' putative deficits for dynamic stimulus detection was restricted to short duration stimuli or, alternatively, whether any group differences might reflect more general deficits in motion integration independent of stimulus duration. If their sensitivity was limited by the ability to detect short duration visual events then dyslexics should demonstrate increased coherent motion thresholds if stimulus duration was shortened. Alternatively, a generalised elevation of dyslexics' motion thresholds that was independent of stimulus duration would provide evidence for a sensitivity deficit mediated by dyslexics' poorer integration of motion signals.

In Experiment 2 we varied the dot density of the RDK displays to examine whether dyslexics were less efficient than controls at extracting the spatially diffuse motion information that occurs when dot density is decreased. We varied the number of the stimulus elements while keeping the stimulus panel size constant. This manipulation had the effect of amplifying the motion and luminance energy in the stimulus by pro- 
portionally increasing both the average number of target and noise dots per degree of visual angle. The motion signal available within the receptive field of each motion detector was therefore increased, although the overall ratio of signal to noise dots remained constant. If dyslexics were less efficient at extracting the motion signal impinging on motion detectors, their coherent motion sensitivity would be reduced compared to controls, especially at low and intermediate dot densities where the motion information was more sparse.

\section{Methods}

\subsection{Subjects}

All our methods conformed to the tenets of the Declaration of Helsinki and also had local ethics committee approval. The subjects were 10 adult dyslexics [mean age $=25.2$ years (range 19-49)] and 10 adult controls [mean age $=22.2$ years (range 20-31)]. All were native British-English speakers. Clinical or educational psychologists had previously diagnosed each of the dyslexics as reading disabled on the basis of significant discrepancies between their literacy skills and their other cognitive abilities. They were not selected on any basis other than their having a history of dyslexia. We also assessed all of our subjects' cognitive and literacy skills at the time of testing. The cognitive measures comprised the Block Design, Picture

\section{Table 1}

Psychometric statistics for the 10 developmental dyslexic and 10 control subjects ${ }^{\mathrm{a}}$

\begin{tabular}{llll}
\hline $\begin{array}{l}\text { Measure } \\
\text { (unit) }\end{array}$ & $\begin{array}{l}\text { Control mean } \\
\text { (SEM) }\end{array}$ & $\begin{array}{l}\text { Dyslexic mean } \\
\text { (SEM) }\end{array}$ & Significance level \\
\hline Age (years) & $22.2(1.1)$ & $25.2(2.8)$ & n.s. \\
WAIS-R BD (SS) & $13.8(0.5)$ & $16.0(0.9)$ & n.s. \\
WAIS-R PA (SS) & $11.6(1.0)$ & $13.2(0.6)$ & n.s. \\
WAIS-R S (SS) & $13.2(0.6)$ & $14.6(0.8)$ & n.s. \\
WAIS-R V (SS) & $14.4(0.5)$ & $13.2(0.6)$ & n.s. \\
WAIS-R AVG (SS) & $13.3(0.4)$ & $14.2(0.6)$ & n.s. \\
WRAT Read (SS) $^{*}$ & $13.5(0.2)$ & $10.8(0.7)$ & $p<0.01$ \\
WRAT Spell (SS) & $13.5(0.2)$ & $9.8(0.8)$ & $p<0.01$ \\
\hline
\end{tabular}

\footnotetext{
${ }^{a}$ The data are presented as group means with standard errors (SEMs) in parentheses. Significance values were obtained using independent groups $t$-tests. n.s. denotes a two-tailed $t$-score with a probability greater than 0.05 . Asterisks $\left(^{*}\right)$ denote that the standard scores (SS) obtained from the WRAT have been converted to an equivalent score with a mean of 10 and a standard deviation of three in order to facilitate comparison with the WAIS-R measures. WAISR: Wechsler adult intelligence scales-revised; BD: Block Design; PA: Picture Arrangement; S: Similarities; V: Vocabulary; AVG: Average of BD, PA, S; WRAT: Wide Range Achievement Test; Read: Reading subscale; Spell: Spelling subscale.
}

Arrangement, Similarities and Vocabulary subscales of the Wechsler Intelligence Scales (revised) (WAIS-R) [62]. Reading and spelling ability were assessed by the Wide Range Achievement Test (WRAT) [24]. At the time of testing, all of the dyslexic subjects had a single word reading and spelling discrepancy at least 1.3 standard deviations below their average performance on the standardised WAIS-R measures. None of the controls had such a discrepancy and the two groups did not differ significantly on any of the WAIS-R measures. The psychometric data for each group are shown in Table 1.

\subsection{Stimuli}

The standard RDK stimuli consisted of 150 high luminance $\left(131.0 \mathrm{~cd} / \mathrm{m}^{2}\right)$, white dots each subtending $0.05 \times 0.09$ retinal degrees, presented on the black background $\left(0.2 \mathrm{~cd} / \mathrm{m}^{2}\right)$ of a 17 inch CRT monitor display (Gateway Vivitron 1776); giving a space averaged luminance of $2.6 \mathrm{~cd} / \mathrm{m}^{2}$. At a constant viewing distance of $57 \mathrm{~cm}$ the stimulus area subtended $7 \times 7^{\circ}$ centred on the fovea. Viewing was binocular throughout the testing. Michelson contrast $\left[\left(\mathrm{L}_{\max }-\mathrm{L}_{\min }\right) /\left(\mathrm{L}_{\max }+\mathrm{L}_{\min }\right)\right]$ between the luminance of the stimulus dots and the luminance of the background was held at $99.7 \%$ and presentation occurred in a dark room (surrounding space average luminance $\left.=0.8 \mathrm{~cd} / \mathrm{m}^{2}\right)$. The proportion of coherently moving dots (angular velocity $=8.8^{\circ} / \mathrm{s}$ ) within a given software animation frame (duration $=50$ ms) was varied to the subject's detection threshold by custom software written for PCs. Each dot had a limited lifetime of four animation frames $(200 \mathrm{~ms})$ after which it would disappear and then reappear at a random location within the stimulus patch. The noise dots had the same displacements and limited lifetimes as the coherent target dots but moved in a Brownian manner prior to being plotted at random locations within the stimulus patch at the end of their lives.

\subsection{Procedure}

The subjects were asked to fixate a cross that preceded the presentation of the stimulus and remained at the centre of the RDK for its entire duration. After each presentation the subject was asked to report the direction in which coherent motion was perceived, guessing if necessary. The proportion of coherently moving dots, which flowed either rightward or leftward, was altered to the subject's motion threshold by a 1-up 1-down, two alternative, forced choice, single staircase procedure [27]. A correct response was followed by a reduction in the coherence signal by $1 \mathrm{~dB}$ whereas an incorrect answer led to the signal being increased by $3 \mathrm{~dB}$. Motion coherence thresholds were defined as the geometric mean of the last eight of 10 
staircase reversals. Thresholds were corrected for finite dot lifetimes so that in the case where all dots were moving coherently, and each had a lifetime of four frames, this was described as $75 \%$ coherence. Each test comprised three staircases and overall subject thresholds were defined as the arithmetic mean of these estimates. The subjects were given audio feedback indicating correct or incorrect responses. Within each of the experiments, the order of the various levels of the independent variable (stimulus duration and dot density) was counterbalanced between individuals.

\subsection{Experiment 1: effect of stimulus duration}

The control and dyslexic subjects viewed the RDK stimuli for stimulus durations of 4, 9, 18 and 36 animation frames, which correspond to 200, 451, 902 and $1804 \mathrm{~ms}$, respectively. Two dot sizes were used: small dots $\left(0.05 \times 0.09^{\circ}\right)$ and large dots $\left(0.10 \times 0.13^{\circ}\right)$.

\subsection{Experiment 2: effect of dot density}

The $7 \times 7^{\circ}$ stimulus panel was modified to contain either $75,150,300$ or 600 dots. This corresponds to an average dot density of $1.5,3.1,6.1$, and $12.2 \mathrm{dots} / \mathrm{deg}^{2}$, respectively. The stimulus duration was held constant at $902 \mathrm{~ms}$.
3. Results

\subsection{Effect of stimulus duration on coherent motion detection}

A 2(group) $\times 4$ (stimulus duration) mixed factors Analysis of Variance (ANOVA) with group as the between subjects factor and stimulus duration as the within subjects factor was performed on the mean coherent motion data of the subjects for both dot size conditions. For the small dots there were significant main effects both for stimulus duration $[F(3,54)=11.7, p \leq 0.001]$ and group $[F(1,18)=9.9$, $p \leq 0.01]$. However, the interaction between group and stimulus duration was not significant $[F(3,54)=0.99$, $p=0.41]$. A similar pattern of results was found for the large dots. There were significant main effects both for stimulus duration $[F(3,54)=7.0, p \leq 0.001]$ and for group $[F(1,18)=11.5, p \leq 0.001]$ but no interaction between group and stimulus duration $[F(3,54)=0.28$, $p=0.84]$. Fig. 1 presents these results graphically. Comparison of group means by independent groups $t$ tests, with degrees of freedom corrected for variance inhomogeneity when necessary, showed that the dyslexic group was significantly less sensitive than the controls for each of the stimulus durations tested in both dot size conditions (independent groups $t$-test: $\min$ $t(18)=2.4, \quad p=0.026$, two-tailed; $\max t(18)=3.3$, $p=0.004$; two-tailed). There were also no systematic

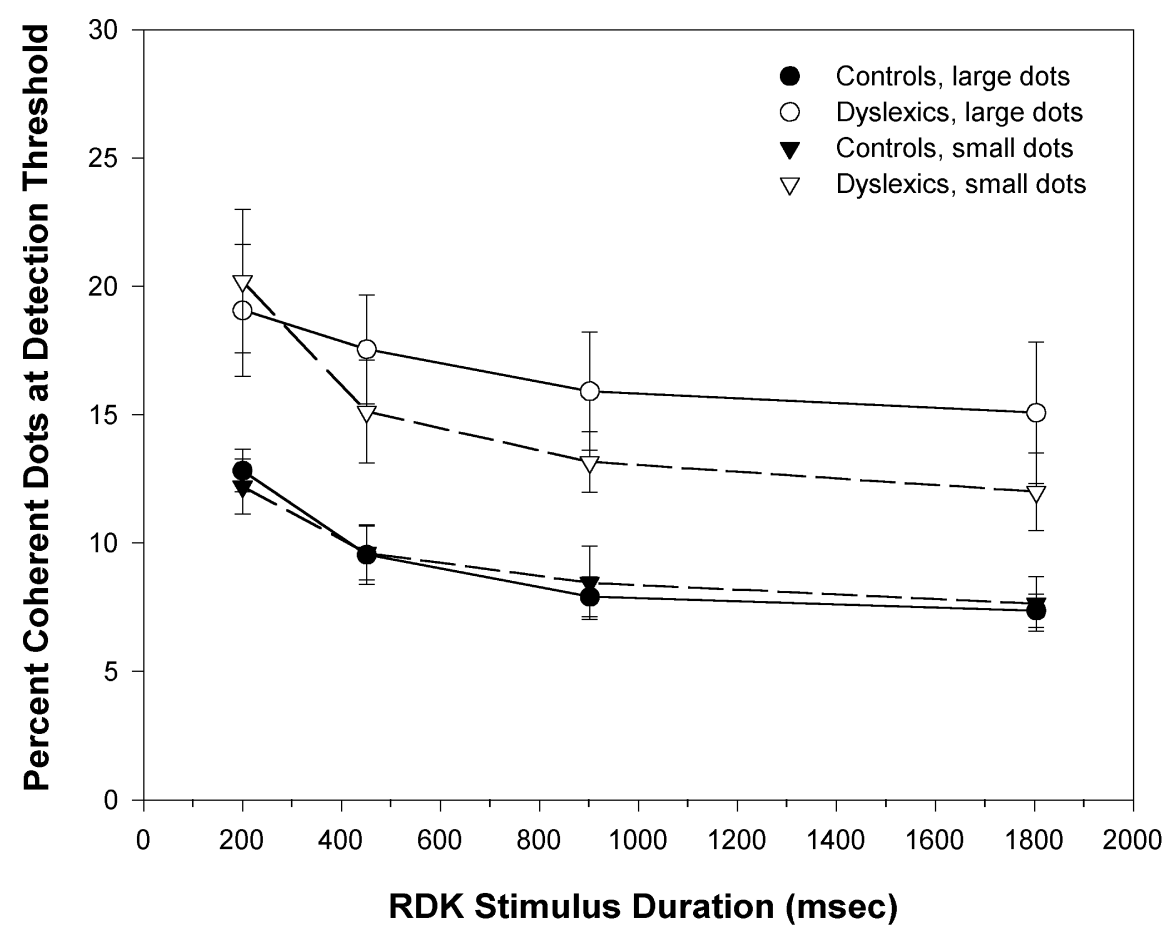

Fig. 1. Thresholds for detecting coherent motion in RDK stimuli, for the 10 control (filled symbols) and 10 dyslexic (empty symbols) subjects, as a function of stimulus duration (msecs) and dot size (large dots: circles/solid lines; small dots: triangles/hatched lines). Error bars correspond to \pm 1 standard error of the mean (SEM) bounding each group's average threshold at each stimulus duration and dot size. 
(a)

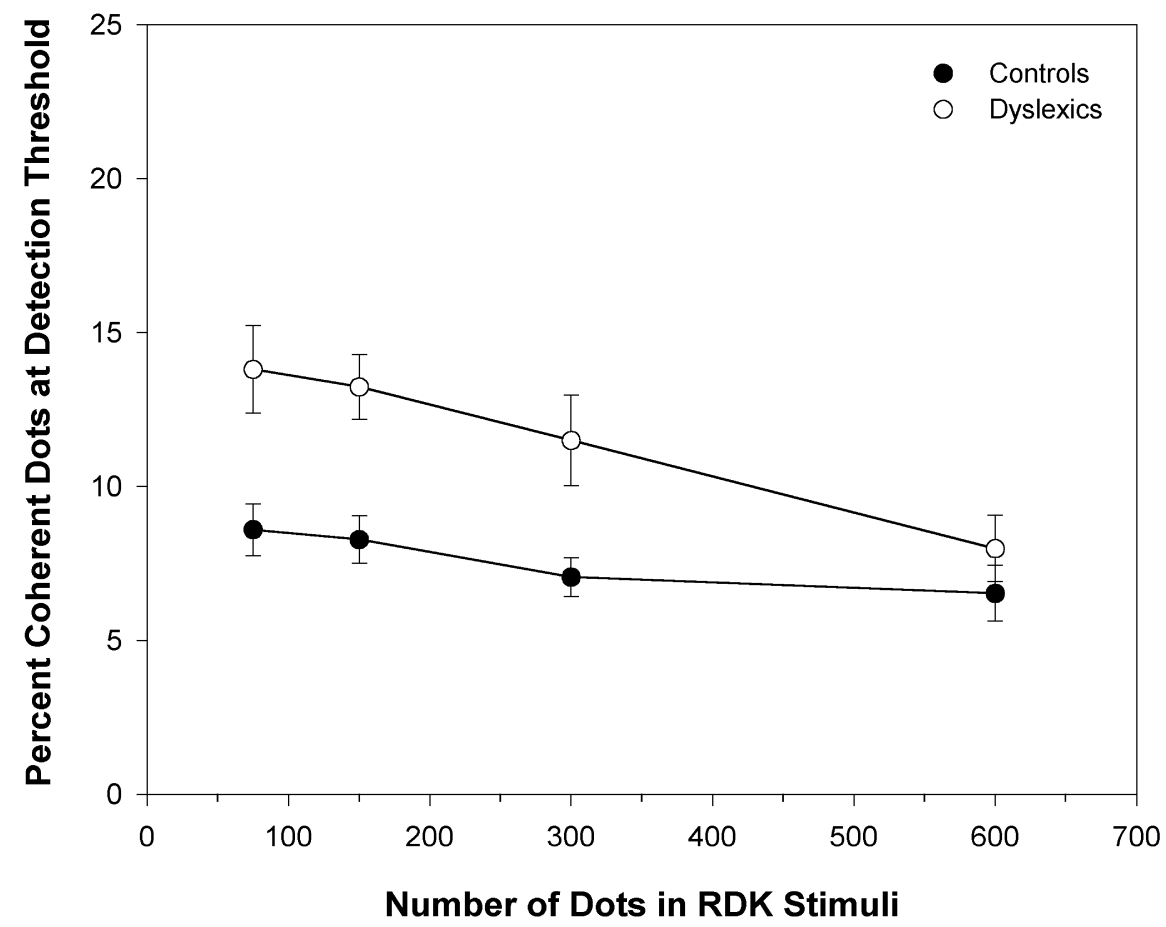

(b)

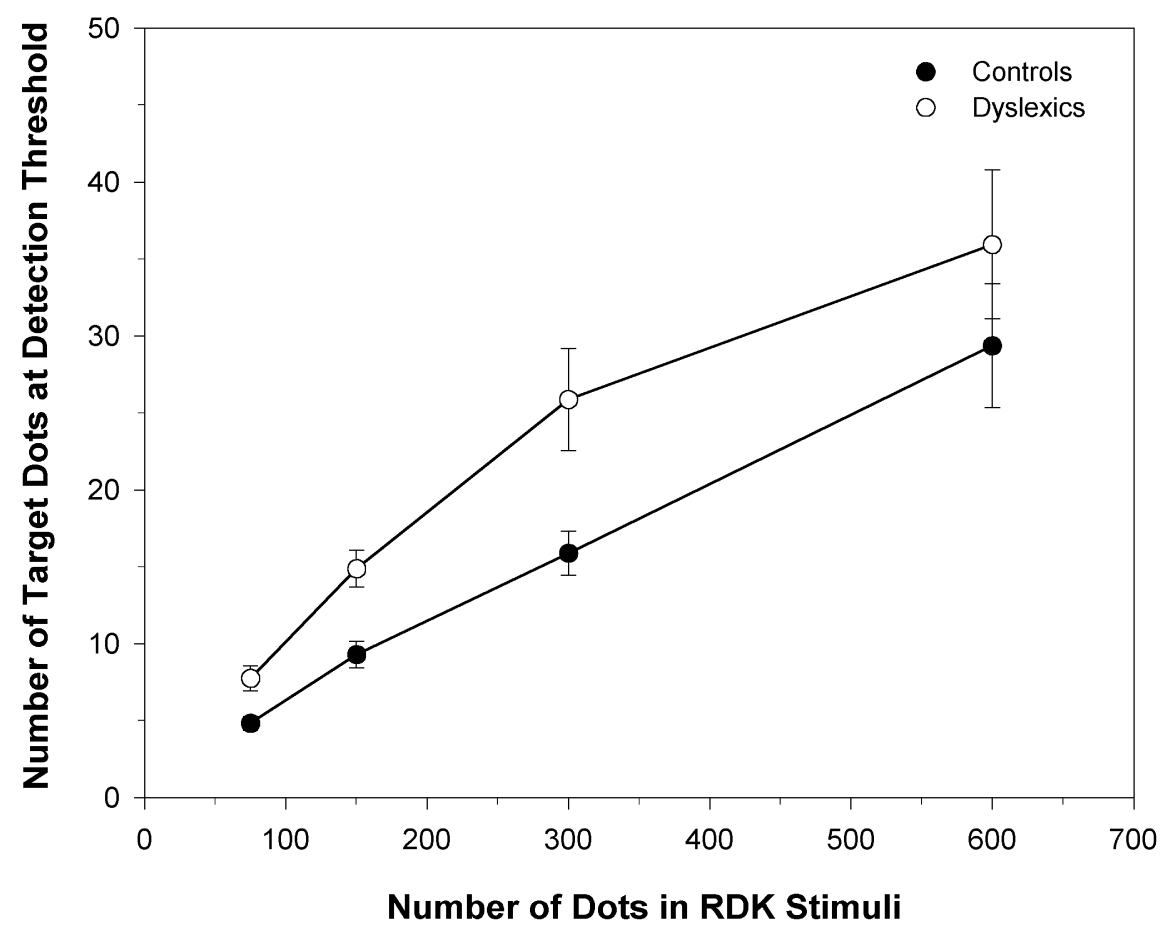

Fig. 2. (a) Thresholds for detecting coherent motion in RDK stimuli for the 10 control (filled symbols) and 10 dyslexic (empty symbols) subjects. Detection thresholds are expressed as the percentage of coherent target dots necessary for coherent motion detection. Error bars correspond to \pm 1 standard error of the mean (SEM) bounding each group's average motion detection threshold. (b) Thresholds for detecting coherent motion in RDK stimuli for the 10 control (filled symbols) and 10 dyslexic (empty symbols) subjects. The data are the same as in (a) but here the detection threshold is plotted as the number of target dots necessary for coherent motion detection. Error bars correspond to \pm 1 standard error of the mean (SEM) bounding each group's average motion detection threshold. 
differences between individual subject's performance for a given stimulus duration between the two dot size conditions (paired sample $t$-test: $\max t(19)=1.37$, $p=0.19$ ). This is consistent with previous results that showed that maximal displacement thresholds for coherent motion are unaffected by element size up to about 10 arc $\min \left(0.17^{\circ}\right)$ [3]. In general coherent motion thresholds increased (i.e., sensitivity decreased) similarly for both groups as stimulus duration decreased, but the magnitude of the group difference was largely independent of stimulus duration and dot size. In both conditions the mean sensitivity of each group appeared to reach asymptote in the $902 \mathrm{~ms}$ condition.

\subsection{Effect of dot density on coherent motion detection}

Fig. 2 shows the effect of varying dot density on the coherent motion thresholds of both groups. The same data is plotted both as percent coherent motion (Fig. 2a) and as threshold dot number (Fig. 2b) at each dot density. Percent coherent motion refers to the threshold ratio of signal to noise dots (see Section 2) whereas threshold dot number refers to the total number of signal dots necessary for detection in each condition. A 2 (group) $\times 4$ (dot density) mixed factors ANOVA, with group as a between subjects factor and dot density as a within subjects factor, revealed significant main effects for group $[F(1,18)=11.9, p<0.01]$ and for dot density $[F(3,54)=10.9, p<0.001]$, but not a significant interaction effect $[F(3,54)=2.6$, $p=0.063]$. Independent groups $t$-tests showed that the dyslexic group was significantly less sensitive than the controls in each condition (independent groups $t$ : $\leq 0.02$, two-tailed) except at the highest dot density of $12.2 \mathrm{dots} / \mathrm{deg}^{2}$. In this condition the dyslexics performance was not significantly impaired relative to controls $[t(18)=1.04, p=0.31$, two-tailed $]$.

\section{Discussion}

Our main result was that dyslexics' sensitivity to coherent motion was significantly reduced relative to controls in both experiments. This finding is consistent with previous studies $[10,16,43,52,58,64]$ and provides additional detailed support for the hypothesis that developmental dyslexia is associated with poor global motion processing. In both experiments the differences between groups were largely independent of changes in the temporal and spatial parameters of the RDKs, demonstrating that the coherent motion deficit in dyslexia is a robust effect.

In Experiment 1 (see Fig. 1) both groups' thresholds were highest at the shorter stimulus durations (minimum $200 \mathrm{~ms}$ ) and decreased to asymptote around 902 ms. The dyslexic group, however, was less sensitive to motion than the controls at each of the stimulus durations tested. Consistent with our previous estimate [58], the magnitude of this group difference was approximately $-2.4 \mathrm{~dB}$ (averaged over the 4 duration conditions). This effect size remained relatively invariant when stimulus duration was increased, as shown by the lack of a significant interaction between group and stimulus duration. This result refutes the hypothesis that dyslexics' visual impairments, however mild, are restricted to short duration visual stimuli. Most previous studies have used static stimuli whose duration was restricted rather than dynamic stimuli such as our RDKs (see [17] for review). The group differences we found for a dynamic visual motion stimulus were independent of stimulus duration. Hence, it seems that our dyslexic sample was less able to detect a continuously changing motion signal, rather than being unable to detect stimuli with restricted durations of presentation.

The software animation frame duration of our RDK stimuli was held constant at $50 \mathrm{~ms}$, and increases in stimulus duration were achieved by increasing the number of animation frames rather than frame duration. A constant dot lifetime of four frames $(200 \mathrm{~ms})$ thus prevented subjects from being able to track the motion of a single dot. The dyslexics' deficits for detecting these RDK stimuli are therefore more likely to result from poor physiological summation and integration of motion signals rather than a failure to visually track motion trajectories at longer stimulus durations. From pilot studies we have determined that it is unlikely that between group differences in understanding task demands or the effects of involuntary eye movements confounded these results. We made sure that both the dyslexic and control groups were able to follow the experimental protocol, and that they could maintain visual fixation for the full duration of stimulus presentation. Any systematic confounding effects would also result in a significant group by stimulus duration interaction, which was not evident. The group differences we found were as large at the higher stimulus durations where eye movements would have been more likely, as they were for the lowest stimulus duration $(200 \mathrm{~ms})$ where eye movements were less probable (Fig. 1). The lack of a group by stimulus duration interaction effect also indicates that it is unlikely that attentional differences between groups, at least over the range of $200-1800 \mathrm{~ms}$, were responsible for the sensitivity differences observed.

In Experiment 2 we varied the number of the dots within the stimulus patch over the range 75-600 dots. This increased dot density from a minimum of two to a maximum of 12 elements per square degree of visual angle in the area subtended by our stimulus. This manipulation had the effect of increasing the total motion 
signal within our stimulus RDKs, with a proportional increase both in the number of signal and noise dots. Examination of Fig. 2a shows that the controls were already at or near a threshold asymptote for coherent motion detection in the 150 dot RDKs and that increasing the dot density thereafter had little effect on their coherent motion thresholds. The controls' ability to extract signal from noise was thus unaffected by the total motion energy within the stimulus. This result is consistent with previous work that showed that other threshold measures of motion detection in controls, for example the maximum $\left(\mathrm{d}_{\max }\right)$ and minimum displacements $\left(\mathrm{d}_{\text {min }}\right)$ detectable in two-frame RDKs, are little affected by changes in dot density [3].

In contrast to controls, the dyslexics had higher detection thresholds in every condition except at the highest dot density (12 dots/ $\left./ \mathrm{deg}^{2}\right)$ when their performance was nearly equivalent. This poorer sensitivity might result from a difference in their cellular physiology that reduced the effective signal to noise level in their motion detectors. They could have lower response gain, more inherent noise or sparser spatial sampling. In the highest dot density condition the dyslexics could benefit from the additional motion energy afforded by the highest density display to partially alleviate this poor signal to noise ratio. It is not clear whether the sensitivity improvement at high dot densities is achieved by more efficient spatial summation of motion information in extrastriate cells tuned for a particular direction of motion, or by a greater activation of lower level motion detectors that is driven by an increase in the overall luminance of the video display. Over our range of dot densities the space averaged luminance increased from 1.4 to $10 \mathrm{~cd} / \mathrm{m}^{2}$ between our lowest and highest dot density condition and the overall luminance of the visual stimuli employed is an important parameter for isolating Mcells $[26,48]$.

Among other possibilities (see $[11,57,59,63]$ for review), integration of motion information necessary to 'solve' the motion correspondence problem presented by an RDK could be achieved by neural networks of cooperative motion analysers consisting of excitatory and inhibitory synaptic networks of lower level motion detectors [7,42]. Most V5/MT cells show direction of motion selectivity [14,22,36] and many show centre-surround antagonism in which the responses of receptive field centres are enhanced if motion in the surround is opposite to that which is impinging on the centre [59]. Cells within V5/MT are arranged in orderly arrays of columns [1], each containing cells selective for a particular range of motion direction. Columns optimally sensitive to similar motion directions are probably linked by excitatory connections, while others are inhibited. Therefore a cell stimulated by motion information in one direction would facilitate the response of similarly tuned cells, while at the same time inhibiting cells tuned for a different motion vector. Increasing dot density, while not changing the ratio of signal to noise dots, increases the number of target dots moving in one particular direction. Hence, dyslexics' performance may improve because the increased motion signal can accumulate in cells tuned for that particular direction of motion.

Dyslexics' pattern of poor motion sensitivity is consistent with the overall pattern of deficit shown by a patient with bilateral lesions to area MT [4]. Using a stimulus similar to ours, Baker, Hess and Zihl showed that patient LM had profound deficits for detecting coherent motion signals in the presence of both dynamic and static noise, with relative sparing of motion detection in conditions without noise. This result suggested that processing of stimuli with low signal to noise ratio was impaired, possibly because the number of extrastriate neurons that code directional motion information was reduced by the lesions. For both dyslexics' $[10,16,43,52,58]$ and patients with V5/ MT lesions $[4,23,60]$, the consistency and magnitude of their reduced dynamic sensitivity are apparently largest when stimulus detection requires a comparison of outputs from visual filters, as is required for detecting coherent motion. Detection of stimulus parameters such as spatial frequency and flicker is carried out by lower level visual filters, and these are only slightly, though in most cases, significantly impaired in the same groups of dyslexics [10,16,52,58] and in LM [23]. Although our dyslexics' visual deficits are not as profound as those reported for LM, their overall deficits for motion detection are consistent with their having similarly impaired integration of dynamic visual signals. Such evidence supports an extrastriate locus of dyslexics' impaired motion sensitivity, probably in V5/ MT and adjacent motion processing areas.

How poor motion processing occurs in dyslexia is unknown, although it may result from the impaired development of magnocells in both LGN and cortex that has been suggested by previous studies $[18,25,28,44]$. Livingstone et al. [28] reported a $20 \%$ reduction in the area of magnocellular but not parvocellular cells in the lateral geniculate nucleus of dyslexics' brains examined post mortem. Although no such specific differences are found in the cell layers that receive specific inputs from magnocells and parvocells in primary visual cortex [25], Rae et al. [44] have demonstrated that dyslexics have lowered cell density relative to controls in an area subsuming the posterior parietal cortex (PPC), an area receiving a predominant input from magnocells $[38,39,56]$. One hypothesis consistent with both our psychophysical data and these anatomical studies is that dyslexics with visual deficits 
have fewer motion detectors, and therefore undersample spatially diffuse dynamic stimuli such as coherent RDK motion.

\section{Conclusion}

The consistency of dyslexics' motion detection impairment was unaffected by stimulus duration. This demonstrates that the visual deficit is more related to their poor integration of the changes in time that are characteristic of dynamic visual stimuli than to more generalised detection deficits for stimuli with limited presentation durations. The difference in sensitivity between the groups was similarly unaffected by element density except at the highest dot density where dyslexics approached that of controls. We suggest that this improvement is a consequence of the greater motion energy in the denser RDKs, which boosts the effective signal to noise ratio. Such experimental evidence is consistent with an extrastriate locus of their motion processing deficits, perhaps in combination with decreased sensitivity of lower level M-cells in the retino-cortical pathway. It is also consistent with evidence of anomalous cellular anatomy in dyslexics which is most prominent in areas with a predominant visual magnocellular input.

\section{Acknowledgements}

This research was supported by the Wellcome Trust (JBT, JFS) and the MRC-IRC (PCH). We thank Piers Cornelissen, Caroline Witton, Burton Rosner and an anonymous referee for their helpful comments and suggestions. Jonathan Winter also provided invaluable technical support.

\section{References}

[1] Albright T, Desimone R, Gross C. Columnar organization of directionally selective cells in visual area MT of the macaque. Journal of Neurophysiology 1984;51:16-31.

[2] American Psychiatric Association [4th rev. ed.]. In: Diagnostic and statistical manual of mental disorders. Washington DC: APA, 1994. p. 48 [4th rev. ed.].

[3] Baker C, Braddick O. The basis of area and dot number effects in random dot motion perception. Vision Research 1982;22:851-6.

[4] Baker C, Hess R, Zihl J. Residual motion perception in a "motion-blind" patient, assessed with limited lifetime random dot stimuli. Journal of Neuroscience 1991;11:454-61.

[5] Born R, Tootell R. Segregation of global and local motion processing in primate middle temporal visual area. Nature 1992;357:497-9.

[6] Braddick O. Segmentation vs integration in visual motion processing. Trends in Neurosciences 1993;16:263-8.
[7] Braddick O. A short range process in apparent motion. Vision Research 1974;14:519-27.

[8] Bradley L, Bryant P. Categorizing sounds and learning to read - a casual connection. Nature 1983;301:419-21.

[9] Britten K, Shadlen M, Newsome W, Movshon J. The analysis of visual motion: a comparison of neuronal and psychophysical performance. Journal of Neuroscience 1992;12:4745-65.

[10] Cornelissen P, Richardson A, Mason A, Fowler S, Stein J. Contrast sensitivity and coherent motion detection measures at photopic luminance level in dyslexic readers and controls. Vision Research 1995;35:1483-94.

[11] Dawson M. The how and why of what went where in apparent motion: Modeling solutions to the motion correspondence problem. Psychological Review 1991;98:569-603.

[12] Demb J, Boynton G, Heeger D. Brain activation in visual cortex predicts individual differences in reading performance. Proceedings of the National Academy of Sciences (USA) 1997;94:13363-6.

[13] Derrington A, Lennie P. Spatial and temporal contrast sensitivities of neurones in lateral geniculate nucleus of macaque. Journal of Physiology (London) 1984;357:219-40.

[14] Dubner R, Zeki S. Response properties and receptive fields of cells in an anatomically defined region of the superior temporal sulcus in the monkey. Brain Research 1971;35:528-32.

[15] Eden G, Vanmeter J, Rumsey J, Maisog J, Woods R, Zeffiro T. Abnormal processing of visual motion in dyslexia revealed by functional brain imaging. Nature 1996;382:66-9.

[16] Everatt J, Bradshaw M, Hibbard P. Visual processing and dyslexia. Perception 1999;28:243-54.

[17] Farmer M, Klein R. The evidence for a temporal processing deficit linked to dyslexia: A review. Psychonomic Bulletin \& Review 1995;2:460-93.

[18] Galaburda A, Sherman G, Rosen G, Aboitz F, Geschwind N. Developmental dyslexia: Four consecutive cases with cortical anomalies. Archives of Neurology 1985;18:222-33.

[19] Gross K, Rothenburg S, Schottenfeld S, Drake C. Duration thresholds for letter identification in left and right visual fields for normal and reading-disabled children. Neuropsychologia 1978;16:709-15.

[20] Gross-Glenn K, Skottun B, Glenn W, Kushch A, Lingua R, Dunbar M, et al. Contrast sensitivity in dyslexia. Visual Neuroscience 1995;12:153-63.

[21] Hayduk S, Bruck M, Cavanagh P. Low-level visual processing skills of adults and children with dyslexia. Cognitive Neuropsychology 1996;13:975-1015.

[22] Heeger D, Boynton G, Demb J, Seideman E, Newsome W. Motion opponency in visual cortex. Journal of Neuroscience 1999;19:7162-74.

[23] Hess R, Baker C, Zihl J. The "motion-blind" patient: Low-level spatial and temporal filters. Journal of Neuroscience 1989;9:1628-40.

[24] Jastak S, Wilkinson G. Wide-range achievement test. Wilmington, DE: Jastak Associates, 1984 [revised].

[25] Jenner A, Rosen G, Galaburda A. Neuronal asymmetries in primary visual cortex of dyslexic and nondyslexic brains. Annals of Neurology 1999;46:189-96.

[26] Kaplan E, Lee B, Shapley R. New views of primate retinal function. Progress in Retinal Research 1990;9:273-336.

[27] Kaernbach C. Simple adaptive testing with the weighted updown method. Perception \& Psychophysics 1991;49:227-9.

[28] Livingstone M, Rosen G, Drislane F, Galaburda A. Physiological and anatomical evidence for a magnocellular defect in developmental dyslexia. Proceedings of the National Academy of Sciences (USA) 1991;88:7943-7.

[29] Lovegrove W. Do dyslexics have a visual deficit? In: Wright S, Groner R, editors. Facets of dyslexia and its remediation. Amsterdam: North-Holland, 1993. p. 33-49. 
[30] Lovegrove W, Martin F, Slaghuis W. A theoretical and experimental case for a visual deficit in reading disability. Cognitive Neuropsychology 1986;3:225-67.

[31] Lovegrove W, Martin F, Bowling A, Blackwood M, Badcock D, Paxton S. Contrast sensitivity functions and specific reading disability. Neuropsychologia 1982;20:309-15.

[32] Lovegrove W, Williams M. Visual temporal processing deficits in specific reading disability. In: Willows D, Kruk R, Corcos E, editors. Visual processes in reading and reading disabilities. Hillsdale, NJ: Lawrence Erlbaum, 1993. p. 311-30.

[33] Martin F, Lovegrove W. The effects of field size and luminance on contrast sensitivity differences between specifically reading disabled and normal children. Neuropsychologia 1984;22:72-7.

[34] Martin F, Lovegrove W. Flicker contrast sensitivity in normal and specifically disabled readers. Perception 1987;16:215-21.

[35] Mason A, Cornelissen P, Fowler M, Stein J. Contrast sensitivity, ocular dominance and specific reading disability. Clinical Vision Sciences 1993;8:345-53.

[36] Maunsell J, Van Essen D. Functional properties of neurons in middle temporal visual area of the macaque monkey I: Selectivity for stimulus direction, speed, and orientation. Journal of Neurophysiology 1983;49:1127-47.

[37] Merigan W, Maunsell J. How parallel are the primate visual pathways? Annual Review of Neuroscience 1993;16:369-402.

[38] Merigan W, Byrne C, Maunsell J. Does motion perception depend upon the magnocellular pathway? Journal of Neuroscience 1991;11:3422-9.

[39] Milner D, Goodale M. The visual brain in action. Oxford: Oxford University Press, 1995.

[40] Newsome W, Britten K, Movshon J. Neuronal correlates of a perceptual decision. Nature 1989;341:52-4.

[41] Newsome W, Paré E. A selective impairment of motion processing following lesions of the middle temporal visual area (MT). Journal of Neuroscience 1988;8:2201-11.

[42] Newsome W, Wurtz R, Dursteler M. Deficits in visual motion processing following ibotenic acid lesions of the middle temporal area of the macaque monkey. Journal of Neuroscience 1985;5:825-40.

[43] Raymond J, Sorenson R. Visual motion perception in children with dyslexia: normal detection but abnormal integration. Visual Cognition 1998;5:389-404.

[44] Rae C, Lee M, Dixon R, Blamire A, Thompson C, Styles P, et al. Metabolic abnormalities in developmental dyslexia detected by ${ }^{1} \mathrm{H}$ magnetic resonance spectroscopy. The Lancet 1998;351:1849-52.

[45] Riddoch G. Dissociation of visual perceptions due to occipital injuries with special reference to appreciation of movement. Brain 1971;40:15-7.

[46] Ruddock K. Visual search in dyslexia. In: Stein J, editor. Vision and visual dysfunction: vision and visual dyslexia, vol. 13. Boca Raton, FL: CRC Press, 1991. p. 58-83.

[47] Rutter M, Yule W. The concept of specific reading retardation. Journal of Child Psychology and Psychiatry 1975;16:181-97.

[48] Shapley R. Visual sensitivity and parallel retinocortical channels. Annual Review of Psychology 1990;41:635-58.

[49] Shaywitz S. Current concepts — dyslexia. New England Journal of Medicine 1998;338:307-12.
[50] Shaywitz S, Shaywitz B, Fletcher J, Escobar M. Prevalence of reading disability in boys and girls: results of the Connecticut longitudinal study. Journal of the American Medical Association 1990;264:998-1002.

[51] Siegel R, Andersen R. Perception of three-dimensional structure from motion in monkey and man. Nature 1988;331:259-67.

[52] Slaghuis W, Ryan J. Spatio-temporal contrast sensitivity, coherent motion, and visible persistence in developmental dyslexia. Vision Research 1999;39:651-68.

[53] Snowden R, Braddick O. Extension of displacement limits in multiple exposure sequences of apparent motion. Vision Research 1989;29:1777-87.

[54] Stanovich K, Siegel L, Gottardo A. Progress in the search for dyslexia subtypes. In: Hulme C, Snowling M, editors. Dyslexia: biology, cognition and intervention. London: Whurr, 1997. p. 108-30.

[55] Stanovich K. Explaining the differences between the dyslexic and the garden-variety poor reader: the phonological core, variable-difference model. Journal of Learning Disabilities 1988;21:590-612.

[56] Stein J, Walsh V. To see but not to read; the magnocellular theory of dyslexia. Trends in Neurosciences 1997;20:147-52.

[57] Stoner G, Albright T. Visual motion integration: A neurophysiological and psychophysical perspective. In: Smith A, Snowden R, editors. Visual detection of motion. London: Academic Press, 1994. p. 253-90.

[58] Talcott J, Hansen P, Willis-Owen C, McKinnell I, Richardson A, Stein J. Visual magnocellular impairment in adult developmental dyslexics. Neuro-Ophthalmology 1998;20:187-201.

[59] Tanaka K. Representation of visual motion in the extrastriate visual cortex. In: Watanabe T, editor. High-level motion processing: computational, neurobiological, and psychophysical perspectives. MIT Press, 1998. p. 295-315.

[60] Vaina L, Grywacz N, LeMay M, Bienfang D, Wolpow E. Perception of motion discontinuities in patients with selective motion deficits. In: Watanabe T, editor. High-level motion processing: computational, neurobiological, and psychophysical perspectives. MIT Press, 1998. p. 213-47.

[61] Walther-Müller P. Is there a deficit of early vision in dyslexia? Perception 1995;24:919-36.

[62] Wechsler D. Wechsler adult intelligence scales. San Antonio, TXP: Psychological Corp, Harcourt Brace Jovanovich, 1981 [revised].

[63] Williams D, Brannan J. Spatial integration of local motion signals. In: Smith A, Snowden R, editors. Visual detection of motion. London: Academic Press, 1994. p. 291-303.

[64] Witton C, Talcott J, Hansen P, Richardson P, Griffiths T, Rees A, Stein J, Green G. Sensitivity to dynamic auditory and visual stimuli predicts nonword reading ability in both dyslexic and normal readers. Current Biology 1998;8:791-7.

[65] Zeki S. Cerebral akinetopsia (visual motion blindness). A review. Brain 1991;114:811-24.

[66] Zihl J, von Cramon D, Mai A. Selective disturbance of movement vision after bilateral brain damage. Brain 1983;106:31340. 